\title{
Leveraging Expertise from Community Resources to Improve the Role of the Pharmacist in HIV Testing and Counseling
}

\author{
Edgar S. Diaz-Cruz, PhD ${ }^{1}$; Sara Thompson, PharmD²; Mary Hawkins, MBA ${ }^{3}$; Riguin Zengotita-Borges, PharmD ${ }^{1}$; \\ Kathryn Jefferson, PharmD ${ }^{1}$ \\ ${ }^{1}$ Department of Pharmaceutical Social \& Administrative Sciences, College of Pharmacy, Belmont University; \\ ${ }^{2}$ School of Pharmacy, College of Health Professions, North Dakota State University; ${ }^{3}$ Department of HIV Prevention, Nashville CARES
}

\begin{abstract}
The human immunodeficiency virus (HIV) epidemic continues to be a major global public health issue. Moreover, disparities continue to persist in HIV among racial and ethnic minority populations, with the highest rates of new diagnoses in Black/African American and Hispanic/Latino men who have sex with men in the United States. Pharmacists are one of the most accessible and trusted health care professionals. Therefore, it is imperative that student pharmacists are educated on culturally-competent HIV testing and risk behaviors counseling. This study describes the development of a partnership between a pharmacy school and a community-based organization to offer an HIV counseling and testing training program to help develop skills in delivering HIV testing services. The HIV counseling and testing training program contains learning modules that provide a wide array of in-depth information about HIV patient care in the community. The partnership allows for the enjoyment of a myriad of benefits for students, the pharmacy program, the communitybased organization, and the public health of the community-at-large. Students feel more prepared and comfortable working with patients in discussing HIV transmission risk factors and test results as a result of this training. Such partnerships support the pharmacist's role in the public health arena. A successful and durable relationship between a community partner and a school of pharmacy is a feasible strategy for pharmacy progress in public health.
\end{abstract}

Keywords: Human immunodeficiency virus, public health, community engagement, HIV testing

\section{Introduction}

The human immunodeficiency virus (HIV) epidemic continues to be a major public health crisis in the United States (US). Approximately 1.2 million people are currently living with HIV with 38,000 new diagnoses in 2018, the majority of which occurred in the South of the US. ${ }^{1}$ The overall rate of new HIV diagnoses in the state of Tennessee in 2018 was 11.3 per 100,000 persons, with the highest rates concentrated in the metropolitan areas of Memphis/Shelby County (32.9 per 100,000 persons) and Nashville/Davidson County (18.4 per 100,000 persons). ${ }^{1}$ There are striking health disparities associated among Blacks/African Americans and Hispanics/Latinos who are disproportionately affected by HIV. Rates among these groups are 39.2 and 16.4 per 100,000 respectively, compared to 4.8 in Whites. ${ }^{1}$ Furthermore, in 2018, $69 \%$ of new HIV diagnoses occurred in men who have sex with men (MSM) compared to $24 \%$ of new diagnoses in heterosexuals. ${ }^{2}$ Early diagnosis of HIV is critical for optimizing treatment, reducing transmission, and improving patient outcomes. An estimated $14 \%$ of people living with HIV are unaware of their status. ${ }^{3}$ In spite of this, patients may avoid routine screenings due to lack of knowledge/awareness, perceived low risk for HIV infection, not having access to a health care provider in the past year, among others. ${ }^{4}$ Studies

Corresponding author: Edgar S. Diaz-Cruz, PhD

Belmont University College of Pharmacy

1900 Belmont Boulevard, Nashville, TN 37212

Email: edgar.diaz-cruz@belmont.edu

Phone: 615-460-6532; Fax: 615-460-6537 have shown that cultural factors such as stigma, homophobia, and discrimination prevent patients from receiving HIV testing, decrease the likelihood that carriers will disclose their results, reduce adherence to treatment, reduce the likelihood of viral suppression, increase transmission rates, and decrease quality of life. ${ }^{5}$ Furthermore, research demonstrates that implicit racial/ethnic bias among well-intentioned health care professionals continues to contribute to health disparities in the US. ${ }^{6,7}$

The Federal Drug Administration (FDA) approval of the rapid HIV test for clinical laboratory improvement amendments (CLIA)-waived point-of-care (POC) for pharmacist administration in pharmacies and for sale as self-tests for at home use provided a convenient way to detect anti-HIV antibodies using oral mucosa transudate. ${ }^{8}$ It also created a unique opportunity for pharmacists to provide quick, confidential HIV testing, as well as counseling to reduce transmission of HIV regardless of status. This is especially key for underserved patients who do not have access to other healthcare. ${ }^{9}$ As student pharmacists are training to become future pharmacy practitioners and agents in the community, it is imperative that these students are educated on POC HIV tests and HIV risk behaviors counseling. A 2016 survey reported that $40.6 \%$ of fourth-year students were not familiar with the POC HIV test. An additional $66.9 \%$ reported they did not feel comfortable counseling patients on its use, and $51.5 \%$ felt that their coursework has not adequately prepared them to counsel patients on POC HIV tests. ${ }^{10}$ Furthermore, less than half of students were aware of resources available specifically for HIV in their community and felt comfortable making patient 
referrals to these resources. In a 2017 study of pharmacy students, only $37 \%$ reported curricular exposure to an in-home HIV test and less than $10 \%$ felt confident in counseling a patient on its use. ${ }^{11}$

A 2020 cross-sectional study of pharmacy schools in the US found HIV education in the curriculum to range between 0.5-60 hours with $78 \%$ of schools reporting fewer than 10 hours devoted to HIV education. ${ }^{12}$ An alarming $41 \%$ of schools dedicated fewer than 5 hours to HIV education. ${ }^{12} \mathrm{~A}$ recent survey of licensed pharmacists found that only $21.7 \%$ were aware of the POC test for HIV. ${ }^{13}$ Nevertheless, the growing public health concern of HIV and persistence in disparities in HIV infection in the US indicates that this is insufficient. Some pharmacy programs offer unique opportunities for HIV training. For example, the University of Minnesota College of Pharmacy offers an Interprofessional Collaborative Practice HIV Care elective course. Medical, nursing, and pharmacy students reported increased confidence related to patient care and 93\% stated that they planned to become involved in HIV care in the future. ${ }^{14}$ Cedarville University School of Pharmacy offers second-year pharmacy students a workshop on HIV testing. A survey found that after training, 97\% were able to correctly collect the sample, interpret the test, and interpret the test, and provide counseling on the results. ${ }^{15}$

Community-based organizations are uniquely positioned to work at the local level to meet community needs, while complementing and extending the reach of public health efforts implemented both at the local and state levels. Communitybased organizations offer unparalleled expertise and resources to help pharmacy programs to not only educate their students, but also better equip them to serve and advocate for the communities they represent. Collaborating with communitybased organizations may reduce financial and time limitations for pharmacy programs and increase student awareness of community resources, while increasing the number of welltrained student volunteers for the community-based organization. Moreover, it is imperative that pharmacists take an active role in public health, especially as they aim to improve the health of marginalized and underserved populations. ${ }^{16,17}$ Beyond the introduction of cultural sensitivity in the classroom, pharmacy programs must provide opportunities for students to engage with key stakeholders in the public health workforce. ${ }^{7}$ The purpose of this paper is to provide guidance on how to establish a collaboration between a pharmacy program and an HIV community-based organization to improve education on HIV testing and counseling in an effort to improve public health through reduced transmission of HIV.

\section{Methods of Collaborative Instructional Model Establishing a Collaboration}

While students at Belmont University College of Pharmacy (BUCOP) receive ample education in HIV pathophysiology, antiretroviral pharmacology and therapeutics in our program, this instructional approach aimed to empower and equip future pharmacists to tackle the HIV epidemic using a comprehensive public health approach. As a result, a community-based organization was selected to ensure use of the most current HIV testing and counseling strategies, provide culturallyappropriate content, and help students understand the real barriers and proven interventions for HIV prevention based on the geographical location of the patient population.

Founded in 1985, Nashville CARES serves more than 50,000 clients annually throughout 17 counties in Middle Tennessee. It provides targeted education, free and confidential HIV testing, and a broad range of comprehensive social services to individuals at-risk for or living with HIV. These include transportation assistance, medical and social services appointment scheduling, dietary assistance, financial assistance, in-home help, copay assistance, case management, educational programs, social groups, early intervention services to link patients to medical HIV care, among others. In an effort to address the disproportionate impact of HIV in Black and Latino MSM, Nashville CARES established the My House community center and partnered with two local organizations (Street Works and Neighborhood Health) to provide health and wellness services that are culturally sensitive for MSM of color.

A collaboration between BUCOP and Nashville CARES was first established in the fall of 2012. Part of a private university that thrives in a student-centered Christian community in Nashville, Tennessee, BUCOP admitted its first class in 2008. In response to the limited amount of HIV testing and counseling education in our curriculum, and the need to increase the number of trained HIV testing and counseling volunteers, both parties agreed to develop a formalized HIV counseling and testing training program. The collaboration is particularly important for the pharmacy program because it offers unique expertise and access to a patient population that is highly impacted by the HIV epidemic. As a result, the collaboration optimizes a liaison between student pharmacists, representing the most accessible health care professional, and community health workers. This innovation promises to cultivate a health care workforce committed to work together to improve access to care and reduce HIV health disparities in the most vulnerable patient populations.

\section{Training Development and Instructional Approach}

A two-day (two 8-hour sessions) HIV counseling and testing training certificate program was developed using the "I Know HIV Prevention Counseling and Testing Training" developed by the Tennessee Department of Health (TDOH) and conducted by the Associate Director of Training, Evaluation and Research at Nashville CARES. ${ }^{18}$ Upon successful completion of the HIV counseling and testing training program, students receive three certificates of completion granted by the TDOH: "I Know HIV Prevention Counseling \& Testing”, OraQuick ADVANCE Rapid HIV-1/2 Antibody Test Device Training, and the INSTI ${ }^{\circledR}$ HIV1/HIV-2 Antibody Test Device Training certificates. 
The inaugural HIV counseling and testing training certificate program was first offered in the spring of 2014 and has since been offered annually. A total of 86 individuals have successfully completed the certificate program and received certification (two faculty members and 84 pharmacy students). The training is limited to 12-14 participants to allow for a more intimate learning environment. The selection process is application-based with the following criteria: current year in pharmacy school, student organization and leadership involvement, previous participation in the AIDS walk, previous volunteer experience at Nashville CARES, and personal statement indicating commitment to HIV prevention. Whenever possible, priority is given to students from diverse racial, ethnic and linguistic backgrounds. This approach has proven effective in enhancing HIV counseling and testing for culturally and linguistically diverse populations. Only students in their first (P1), second (P2), or third (P3) professional year at BUCOP were eligible to apply to participate in the HIV counseling and testing. The training is conducted over two consecutive Saturdays in a group conference room at Nashville CARES. The facilitators deliberately hold the training outside of school property in an effort to allow students to be more open to ask questions and share their life experiences. A faculty member oversees the logistics involved in the training.

The training uses a variety of teaching modalities including traditional lecture instruction, video clips, group discussion, guest speakers, and learning activities. The training units, objectives, and learning activities are detailed in Table 1. Prior to formally starting the training session, students are asked to draw a picture that represents them, share a nickname, a list of personal strengths, and a list of what they hope to learn from the training. Each student is asked to share their thoughts with the rest of the group. Following this ice-breaker activity, the trainer emphasizes the importance of confidentiality and shares ground rules, in addition to offering the opportunity for students to ask additional questions. The training was built on the premise that excellent HIV prevention counselors are selfaware of how their personal biases, cultural background, and HIV stigma can get in the way of providing effective patient care. As a result, the first three units, Introduction to Counseling, Self-awareness, and Behavior Change, allow students to honestly reflect on their weaknesses and draw from their strengths in these areas. The next two units, Risk Assessment and Pre-Exposure Prophylaxis (PrEP) and Condoms, teach pharmacy students to recognize HIV risk behaviors, and to counsel patients on two of the most effective HIV prevention strategies (PrEP and condom use). Students are instructed to work through a series of case studies in which they must recognize the risk behavior and counsel the patient on safer behaviors based on a patient's life circumstances.

A substantial amount of time is dedicated to developing expertise in conducting HIV tests and delivering HIV test results. During the hands-on HIV test device training unit, students were instructed on how to properly perform an OraQuick ${ }^{\circledR}$
ADVANCE Rapid HIV-1/2 Antibody Test using an oral fluid sample, and an INSTI $^{\oplus}$ HIV-1/HIV-2 Antibody Test using fingerstick blood. Each student was provided with a testing device for both HIV tests and allowed to practice under supervision with proper biohazard disposal protocols in place. Students were instructed on proper technique to obtain patient samples and device handling. During the Test Results unit, students observed a video-clip of how to properly deliver an HIV negative and HIV positive test result. Students were instructed to write down how they would communicate both results to a patient, and paired up with another student to deliver a randomly assigned OraQuick ADVANCE Rapid HIV-1/2 Antibody Test result (the three possibilities were nonreactive, reactive, and invalid). The next two units, Challenging Situations and Emotional Responses, were dedicated to help students prepare mentally to face challenging situations and the emotional response of patients who receive an HIV test result. As an HIV positive test result can be a life-changing experience for patients, students were instructed on the WEATHER approach to counseling. The approach focuses on (W) watching patients verbal and non-verbal expressions, (E) eliciting emotions to allow patients to express what they are feeling, (A) asking about concerns and fears, $(T)$ treating patients' concerns and fears as normal, $(\mathrm{H})$ helping with hope to ensure patients know they have autonomy over a difficult situation, (E) empowering patients, and (R) relating to HIV to reaffirm with empathy and hope. Understanding that an HIV positive test result needs to be followed up with appropriate linkage to HIV care, students were instructed on the Nashville CARES model of intervention to improve linkage to care. Students learned how to work with early intervention services specialists and case managers to help link newly diagnosed patients to medical HIV care. The training concluded with the Disclosure unit, in which students were instructed on how to support their patients through disclosure and HIV/AIDS Disclosure Law in Tennessee.

Guest speakers from Nashville CARES were strategically selected to bring awareness about important key players in the quest to end the HIV epidemic in Middle Tennessee. A collegeaged speaker was invited to share his perspective on how an HIV diagnosis and relationship with healthcare professionals impacted his life and perspectives. Moreover, students were able to hear from and interact with other community-based health workers, including case managers, early intervention services specialists, PrEP navigators, HIV prevention educators, and public policy and advocacy specialists. This experience allows students to learn about and from other professionals to facilitate effective collaboration and improve health outcomes associated with HIV.

\section{Assessing the Collaborative Instructional Model}

A web-based survey pre- and post-training completion was developed to assess changes in perception of comfort level counseling patients on HIV risk behaviors and the POC HIV test, in addition to perception of knowledge of HIV prevention measures. The survey was optional and all responses were 
collected anonymously. The survey has been administered in the last three training offerings. Six questions assessed basic demographic information (gender, age, race/ethnic identification, current year in pharmacy school, previous academic degrees, and previous special training in HIV testing and counseling outside of pharmacy school coursework). Fourteen questions assessing students' perception of comfort level with counseling patients on risk behaviors for HIV transmission and 11 questions assessing students' perception of preparedness to counsel patients on the POC HIV test and test results were developed as 5-point Likert-type items. The scale ranged from strongly disagree to strongly agree. There were 14 prompts that assessed students' perception of knowledge regarding strategies as measures to prevent HIV using a 4-point Likert-type scale ranging from not knowledgeable to very knowledgeable. Survey statements used in this study can be found in Table 2 .

Each student receives an email explaining the survey topic, informed consent process, anonymous nature of the survey and the estimated length of time needed to complete the survey prior to the start of day one of the training. Students receive an email with the same information at the end of the training on day two. The survey was administered using Qualtrics (Qualtrics, Provo, UT) and was granted exempt status by the Institutional Review Board.

SPSS Statistics 27.0 (IBM Corp, Armonk, NY) was used to analyze the respondent data. As recommended by Harpe, the initial analysis examined the performance of a number of survey items as a group. ${ }^{19}$ Three different scales were used to assess students' perception of comfort level with counseling patients on risk behaviors for HIV transmission (construct 1), students' perception of knowledge regarding strategies used as measures to prevent HIV (construct 2), and students' perception of preparedness to counsel patients on the POC HIV test and test results (construct 3 ). As a measure of internal consistency and scale reliability, Cronbach's alpha analysis was used to estimate the reliability of each construct. Nunnally suggests that the level of reliability is determined by how the measure is being used..$^{20}$ Face validity was used for this idea paper. Experts in the field were consulted to evaluate whether the constructs measure what they are intended to measure; however, no statistical analysis of construct validity have been performed. Independent samples $t$-tests were conducted to identify possible differences between pre- and post-training. $P$-value $<0.05$ was considered significant. Overall, 32 students have completed the pre-training survey and 31 have completed the post-training survey for a response rate of $86.4 \%$ and $83.8 \%$, respectively.

\section{Results and Outcomes}

Summary of Demographic Characteristics of the Sample A total of 14 males and 18 females completed the pre-training survey. Seventeen students were between the ages of 20-25, thirteen were between the ages of 26-30, one student was between the ages of 31-35, and one student was $>36$ years of age. Of the 32 students, 15 identified as Caucasian, seven identified as African American/Black, five identified as Hispanic/Latinx, and five identified as Asian. Twenty students were P1, 9 were P2, and 3 were P3. Sixteen students had a bachelor's degree, eight had an associate's degree, one had a doctoral degree, and seven had no previous academic degrees.

\section{Comfort Level with Counseling on Risk Behaviors for HIV Transmission}

Statements used to measure students' perception of their comfort level with counseling on risk behaviors for HIV transmission are reported in Table 2. The construct showed good reliability (Cronbach's alpha $=0.969$ ). Independent $t$-test analyses demonstrated that students feel significantly more comfortable being able to counsel patients on HIV transmission risk behaviors after completing the HIV testing and counseling training $(p<0.001)$ (Table 3$)$.

\section{Knowledge Regarding Strategies Used as Measures to Prevent HIV}

Statements used to measure students' perception of their knowledge regarding strategies used as measures to prevent HIV transmission are reported in Table 2. The construct showed good reliability (Cronbach's alpha $=0.878$ ). Independent $t$-test analyses showed that students feel significantly more knowledgeable regarding strategies that patients can use to prevent HIV infection $(p<0.001)$ (Table 3$)$.

\section{Preparedness to Counsel Patients on the POC HIV Test and Test Results}

Statements used to measure students' perception of their preparedness to counsel patients on the POC HIV test and the test results are reported in Table 2 . The construct showed good reliability (Cronbach's alpha $=0.974$ ). Independent $t$-test analyses showed that students feel significantly more prepared being able to counsel patients on the OraQuick ADVANCE Rapid HIV-1/2 Antibody Test and its results $(p<0.001)$ (Table 3 ).

\section{Discussion}

A collaboration between a community-based organization and a college of pharmacy was established to create an opportunity to equip student pharmacists to work alongside other health professionals to improve HIV testing and access to care. Evidence from pre- and post-training surveys demonstrates the benefit to student pharmacists as they develop the skillset to offer increased access to care. A partnership between a community-based organization and a pharmacy program allows for a multitude of mutually beneficial advantages that are enjoyed not only by the students in the pharmacy program, but also by other stakeholders. It is important to delineate the benefits from not only the pharmacy program perspective, but also the community partner and the community-at-large in which both entities operate. Benefits were defined and counted as any positive outcome for each stakeholder involved that came as a direct result but were not present before this 
collaboration. Furthermore, it is evident that equipping pharmacy programs with teaching and learning opportunities to help foster effective advocacy and engagement through collaborative models is a priority as stated in the American Association of Colleges of Pharmacy (AACP) 2021-2024 strategic plan. This paper supports AACP strategic priorities by outlining the development of a collaboration between pharmacists and other health professionals that aims to identify and develop evidence-based resources that promote patient well-being.

In addition to the evidence demonstrating increased preparedness in working with HIV testing, additional benefits not directly assessed may be at play. An area that was not heavily assessed but comprises a featured component of the training workshop is communication. In our pharmacy program, communication is formally taught in the P2 year. While P1s are more novice in communication practice, they are allowed to apply for the workshop. This workshop significantly relies on communication skill, and several sections of the curriculum in the workshop were dedicated solely to communication. This focus may increase relative benefit for P1 students. P1s who complete the workshop have an advantage when they partake in the formal communications training in the Doctor of Pharmacy program curriculum. Furthermore, this provides an opportunity for all students to communicate with patients regarding safer sexual and drug use behaviors. While it is possible students will have these opportunities in the experiential component of the pharmacy program, it is unlikely to occur in the didactic setting. The workshop provides a safe space to practice patient-centered counseling skills tailored to the behaviors, life circumstances, and specific needs of the patient being served.

Another benefit that students enjoy in this partnership is the privilege to be trained by individuals who have high levels of cultural competence and who regularly work with marginalized populations. Cultural competence in this regard is related to race/ethnicity, gender identity and sexual orientation, and attitudes, beliefs, and behaviors that are shared by individuals who are at high risk of contracting HIV. Exposure to culturally competent community health workers further develops the students' skillset in working with these populations as they are able to learn from the trainers and observe potential role models of cultural competency.

Students that complete the two-day training receive a tangible deliverable in the form of a certificate from the TDOH. At the end of the HIV counseling and testing training program, students become official and certified HIV testing volunteers for Nashville CARES. As part of this partnership agreement, students are required to provide HIV testing \& counseling services on National HIV Testing Day under the direct supervision of an HIV prevention and education specialist from Nashville CARES. Furthermore, certified students are able to volunteer for HIV testing during the Nashville AIDS Walk,
Nashville Gay Pride Festival, and other community outreach programs. This formal benefit in their professional development gives students a competitive edge when applying for scholarships, internships, post-graduate residencies and fellowships, and full employment.

Beyond the aforementioned benefits to students directly, benefits to the school of pharmacy program are multifold. Schools of pharmacy may consider such a partnership in alignment with co-curricular offerings and may elect to grant co-curricular credit to students who complete the training. Astute experiential education offices may see opportunities to develop introductory pharmacy practice experiences (IPPEs) as they relate to the objectives of the community partnership. More broadly, interprofessional education (IPE) opportunities are offered by way of work with community health workers and public health advocates. Furthermore, student affairs professionals in schools of pharmacy may be able to better support the development of programming in student organizations. At BUCOP, the Student National Pharmaceutical Association (SNPhA) was able to deliver Remember the Ribbon initiatives as a result of the collaboration. The Remember the Ribbon Initiative is dedicated to improving the health and social environment of underserved communities in regards to the HIV/AIDS epidemic through awareness, prevention, and education. ${ }^{21}$ Finally, schools of pharmacy faculty may see avenues via which to demonstrate excellence in professional stewardship. At BUCOP, the faculty liaison who established the original collaboration was honored with the 2016 Professional Stewardship Award. The Professional Stewardship Award recognizes a pharmacist/health care professional who in his or her career has answered the call to service that comes with professional standing in their community. It is important to note that the Office of Student Affairs does not compensate the community organization other than with the provision of food for students during the two-day training. The community organization provides the training as part of their strong commitment to end HIV in the local community.

Benefits to the school of pharmacy also involve additional collaboration springboards in the realm of research productivity. For example, two faculty members from the college serve on a research council representing the community and the pharmacy profession as it relates to HIV prevention. This additional service builds trusting relationships throughout the entire research enterprise to provide health and wellness for MSM and other marginalized populations vulnerable to HIV infection. Furthermore, the faculty member in charge of this collaboration serves in the Community Advisory Board of the AIDS Clinical Trials Group to help integrate community involvement in the AIDS Clinical Trials Units in order to advance HIV/AIDS research.

Though they provide the training and resources for the college of pharmacy, the community organization also benefits from the collaboration. Pharmacy students who complete the 
training go on to provide volunteer hours at various HIV testing events in the local community. They also provide services at fundraisers, such as community walks and food drives. Beyond this certification program, the strong commitment of the college of pharmacy encompasses the direct involvement of the SNPhA Belmont University chapter through the Remember the Ribbon HIV initiative and the BUCOP P(Rx)evention Nashville AIDS walk team. Over the past nine years, this collaboration has positively impacted hundreds of student pharmacists through educational opportunities, hundreds of Middle Tennesseans through HIV testing services, and generated over $\$ 15,000$ for essential support services for people living with HIV.

Similar to faculty in schools of pharmacy, staff members in the community-based organization may have opportunities to demonstrate excellence and dedication through a collaboration. The primary trainer of the workshop received the 2019 Professional Stewardship Award for efforts in collaboration largely due to the HIV counseling and testing program offered to pharmacy students. This award is intended to shine a spotlight on those who serve as role models for our students in their everyday practice and service.

As with any strategic partnership, there is the potential for barriers that may impede optimal success. In its current model, one faculty member is responsible for maintaining the partnership between the community-based organization and the college of pharmacy. Since there is no teaching or administrator credit associated with this certificate program, the faculty member organizes and executes all necessary logistics on a volunteer basis that supports service to the institution. Whereas the initial logistics of developing this partnership took a considerable amount of time, executing this certificate program requires the faculty member to commit about 20 hours per iteration. In order to assure the sustainability of this partnership, programs may choose to increase the number of faculty members involved and/or grant teaching credit. On the community-based organization side, the primary trainer has committed to work a minimum of 16 hours over two consecutive weekends in order to accommodate students' schedules outside of class. The trainer has negotiated compensatory time as a way to account for the overtime hours.

Ultimately, this partnership is committed to train student pharmacists to fulfill their public health responsibilities to the community-at-large through patient-centered HIV counseling. Training a culturally and linguistically competent pharmacy workforce increases the availability of health care services that are sensitive to cultural norms and values accepted by the target population. Patients will benefit from HIV risk-reduction counseling interventions that are culturally competent with respect to race/ethnicity, gender identity, and sexual identity, along with population-specific, culturally based attitudes, beliefs, and behaviors. The community-at-large will benefit from a pharmacist who is equipped to tailor the HIV risk assessment process in a way that is interactive and empathetic focused on helping patients personalize a realistic and feasible HIV risk-reduction plan. Furthermore, patients will benefit from pharmacists who are trained to engage in a holistic approach to HIV prevention and treatment, and who are connected to community resources to which they can be referred regardless of the HIV test result. This creates an ideal opportunity to either link newly diagnosed patients living with HIV to care, or engage in conversations about PrEP or other HIV prevention strategies.

The academy can move forward by identifying partnerships to explore ways to raise pharmacy involvement in public health ventures. There are clearly numerous benefits to all involved parties, including the school of pharmacy that establishes the partnership, the community partner, and the community-atlarge. While it is an investment in time and effort to establish, develop, and maintain a community partnership, with the establishment of clear objectives, the benefits from the relationship far outweigh the cost in faculty time and effort.

\section{Conclusion}

This study describes the process by which a pharmacy program partnered with a community-based organization to establish a training program for student pharmacists to develop skills in delivering HIV testing services. Students feel more prepared and comfortable working with patients in discussing HIV transmission risk factors and test results. Furthermore, the partnership allows for the enjoyment of a myriad of benefits for students, the pharmacy program, and the community-based organization, which ultimately supports the pharmacist's role in public health. A worthwhile strategy for pharmacy progress in public health is the establishment of a durable and mutually beneficial relationship between a community partner and a school of pharmacy.

\section{Acknowledgements: None \\ Funding/support: None \\ Conflicts of Interest: None}

The opinions contained in the paper are those of the authors.

\section{References}

1. HIV in the United States and Dependent Areas. Centers for Disease Control and Prevention website. https://www.cdc.gov/hiv/statistics/overview/ataglan ce.html. Accessed June 8, 2021.

2. HIV and Gay and Bisexual Men. Centers for Disease Control and Prevention website. https://www.cdc.gov/hiv/group/msm/index.html. Accessed June 8, 2021.

3. Basic Statistics. Centers for Disease Control and Prevention website. https://www.cdc.gov/hiv/basics/statistics.html. Accessed June 8, 2021. 
4. Dailey AF, Hoots BE, Hall I, et al. Vital signs: human immunodeficiency virus testing and diagnosis delays - United States. MMWR Morb Mortal Wkly Rep 2017;66:1300-1306.

5. Kay ES, Rice WS, Crockett KB, et al. Experienced HIVrelated stigma in healthcare and community settings: mediated associations with psychosocial and health outcomes. J Acquir Immune Defic Syndr. 2018;77(3):257-263.

6. Hall WJ, Chapman MV, Lee KM, et al. Implicit racial/ethnic bias among health care professionals and its influence on health care outcomes: a systematic review. Am J Public Health. 2015:105(12):60-76.

7. Diaz-Cruz ES. If cultural sensitivity is not enough to reduce health disparities, what will pharmacy education do next? Curr Pharm Teach Learn. 2019;11(5):538-540.

8. OraQuick ADVANCE Rapid HIV-1/2 Antibody Test [package insert]. Bethlehem, PA; OraSure Technologies, Inc.; July 2012. http://www.oraquick.com/assets/base/oraquickfull/p df/8453_02_MultiPager_L.pdf

9. Meyers JE, Farhat D, Guzman A, et al. Pharmacists in HIV prevention: an untapped potential. Am J Public Health. 2019;109:859-861.

10. Diaz-Cruz ES, Bynum LA, Thompson SA. Student pharmacists' perceptions on their preparedness and comfort level in counseling on HIV transmission risk factors and over-the-counter HIV tests. Curr Pharm Teach Learn. 2016;8(6):757-766.

11. Tran A, Stewart AL, Covvey JR. Knowledge, attitudes and beliefs regarding human immunodeficiency virus and in-home testing among a regional sample of student pharmacists. Curr Pharm Teach Learn. 2017;9(6):980-988.

12. Rathburn RC, Durham SH, Farmer KC, et al. Evaluation of human immunodeficiency virus curricular content in schools of pharmacy in the United States. Curr Pharm Teach Learn. 2020;12(8):910-917.
13. Min AC, Andres JL, Grover AB, et al. Pharmacist comfort and awareness of HIV and HCV point of care testing in community settings. Health Promot Pract. 2020;21(5):831-837.

14. Pittenger AL, Goodroad B, Nicol M, et al. Interprofessional education within a pilot HIV-care elective course. Am J Pharm Educ. 2019;83(10):20532058.

15. Staudt AM, Draime JA, Deitschmann N, et al. Training student pharmacists to facilitate human immunodeficiency virus point-of-care testing. Curr Pharm Teach Learn. 2020;12:1245-1251.

16. Palombi I, Kading M, Hayes CJ. The public health pharmacist and the role of the pharmacy curriculum: a call to action. Curr Pharm Teach Learn. 2013;5(5):477-482.

17. McCree DH, Byrd KK, Johnston M, et al. Roles for pharmacists in the "Ending the HIV Epidemic: A Plan for America" initiative. Public Health Rep. 2020;135(5):547-554.

18. Capacity Building Assistance for HIV Prevention Providers. I Know - HIV Prevention Counseling and Testing Training. Tennessee Department of Health website. https://www.tn.gov/health/health-programareas/std/std/hiv-prevention/capacity-buildingassistance-for-hiv-prevention-providers.html. Accessed June 8, 2021.

19. Harpe SE. How to analyze Likert and other rating scale data. Curr Pharm Teach Learn. 2015;7(6):836-850.

20. Nunnally JC. Psychometric Theory. New York, NY: McGraw-Hill; 1978.

21. Remember the Ribbon. Student National Pharmaceutical Association website. https://snpha.org/initiative/rtr. Accessed June 8, 2021. 
Table 1. HIV counseling and testing training units, objectives, and activities

Unit Topic: Objectives
Introduction to counseling:
1. Define the concept of patient-centered counseling
2. Describe the four basic skills used in HIV prevention counseling
3. List the three concepts that guide HIV prevention counseling and discuss
why they are important

Self-awareness:

1. Define key terms associated with the concept of being self-aware

2. Identify three ways that personal belief/judgement as counselors might interfere with effective HIV prevention and behavior change work

Behavior change:

1. Discuss the concept of behavior change and identify the six stages

2. Identify and discuss factors that influence (affect) behavior change

Risk assessment:

1. Define and practice identifying patients' risk behavior and circumstances

2. Discuss and practice ways to help patient select a Safer Goal Behavior and plan Action Steps

PrEP and condoms:

1. Describe what PrEP is and identify good candidates for PrEP

2. Identify resources for PrEP and determine how to refer a patient to a PrEP Navigator

3. Recognize the importance of consistent and correct condom use and be able to educate clients about it

4. Be able to compare and contrast between different condoms

\section{HIV test device training:}

1. Administer an OraQuick ${ }^{\oplus}$ ADVANCE Rapid HIV-1/2 Antibody Test

2. Administer an INSTI ${ }^{\circ}$ HIV-1/HIV-2 Antibody Test

Test results:

1. Discuss the concept of assessing testing readiness

2. Evaluate the best practices for delivering a negative and a positive HIV test result

3. Explain the concept of the "window period" and how it effects an HIV negative test result

Challenging situations:

1. Identify one issue in counseling an HIV-infected patient that poses a challenge

2. Identify basic counseling skills or concepts that will help when dealing with challenging situations

Emotional response:

1. Discuss the concepts of emotional adjustment, and the initial reaction to an HIV positive test

\section{Disclosure:}

1. Define the term disclosure

2. Describe the benefits of disclosure

3. Identify challenges to disclosure

4. Discuss the dangers of non-disclosure

5. Discuss the ways to support disclosure
Unit Activities - Objective

- Wanted: Good counselors - List traits that make a good counselor

- 20 questions - Write ten open- and closed- ended questions

- Values exploration - Explore personal history and attitudes about sexual behaviors

- Agree? Disagree? - Critically reflect on HIV-related "moral" statements

- $\quad$ Town talk - Evaluate the risk of individuals contracting HIV

- The poem of me - Recognize emotions that make you unique

- We have all been there - Behavior you personally changed

- $\quad$ Risk assessment - Identify HIV risk behaviors, action steps, and safer goal behaviors based on scenario

- O.P.R.A.H. and condoms - Demonstrate how to properly use a condom

- $\quad$ To PrEP or Not to PrEP? - Determine if a patient is a good candidate for PrEP and counsel

- Individual device practice - Perform an OraQuick ${ }^{\circledR}$ ADVANCE Rapid HIV-1/2 Antibody Test and an INSTI ${ }^{\circ}$ HIV-1/HIV-2 Antibody Test

- $\quad$ How will I say it? - Practice telling patients their HIV test result (both positive or negative)

- $\quad$ The WEATHER approach - Adapt when dealing with patients' difficult emotions during a counseling session

- Challenging situations - Identify skills and boundaries based on a particular patient counseling scenario

- Imagine for a moment - Empathize with the grieving the loss of hopes and dreams

- To tell or not to tell - List benefits, dangers, barriers, and costs of disclosing one's HIV positive status

$H I V=$ human immunodeficiency virus, $\operatorname{PrEP}=$ pre-exposure prophylaxis 
Table 2. Survey statements used to measure the study's constructs of interest

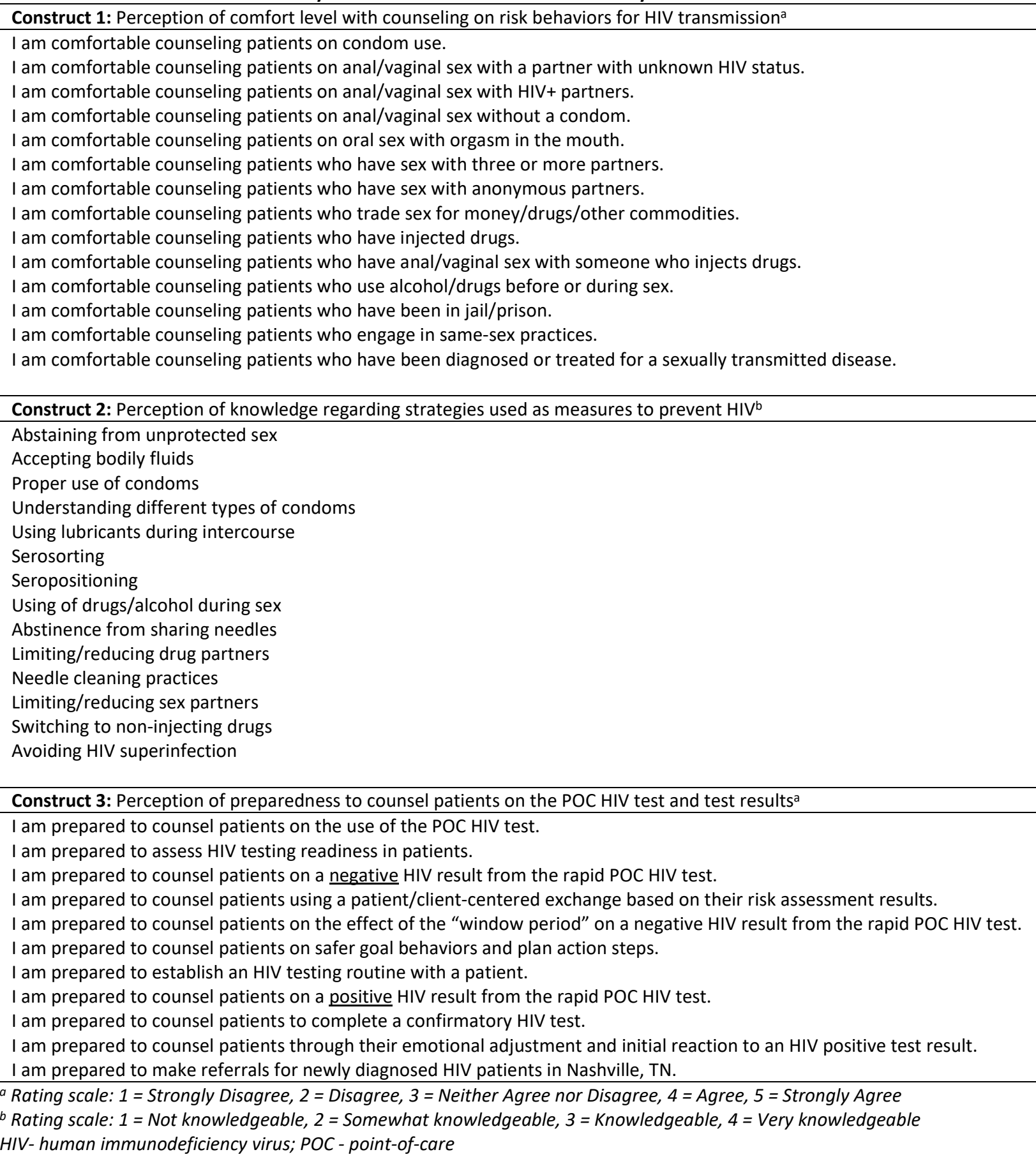

Table 3. Comparison between students pre- and post-HIV testing \& counseling training

\begin{tabular}{|c|c|c|c|}
\hline Construct of interest & Training & Mean \pm SD & Significance \\
\hline Perception of comfort level with counseling on risk behaviors for HIV transmissiona & $\begin{array}{l}\text { Pre }(N=31) \\
\text { Post }(N=31)\end{array}$ & $\begin{array}{l}3.60 \pm 0.82 \\
4.62 \pm 0.46\end{array}$ & $<0.001^{*}$ \\
\hline Perception of knowledge regarding strategies used as measures to prevent $\mathrm{HIV}^{\mathrm{b}}$ & $\begin{array}{l}\text { Pre }(N=32) \\
\text { Post }(N=30)\end{array}$ & $\begin{array}{l}2.27 \pm 0.49 \\
3.65 \pm 0.38\end{array}$ & $<0.001^{*}$ \\
\hline Perception of preparedness to counsel patients on the POC HIV test and test results ${ }^{\mathrm{a}}$ & $\begin{array}{l}\text { Pre }(N=32) \\
\text { Post }(N=30)\end{array}$ & $\begin{array}{l}2.37 \pm 1.03 \\
4.68 \pm 0.41\end{array}$ & $<0.001^{*}$ \\
\hline
\end{tabular}

${ }^{a}$ Rating scale: 1 = Strongly Disagree, $2=$ Disagree, $3=$ Neither Agree nor Disagree, $4=$ Agree, $5=$ Strongly Agree

${ }^{b}$ Rating scale: 1 = Not knowledgeable, 2 = Somewhat knowledgeable, $3=$ Knowledgeable, 4 = Very knowledgeable

* Statistical significance

HIV- human immunodeficiency virus; POC - point-of-care 